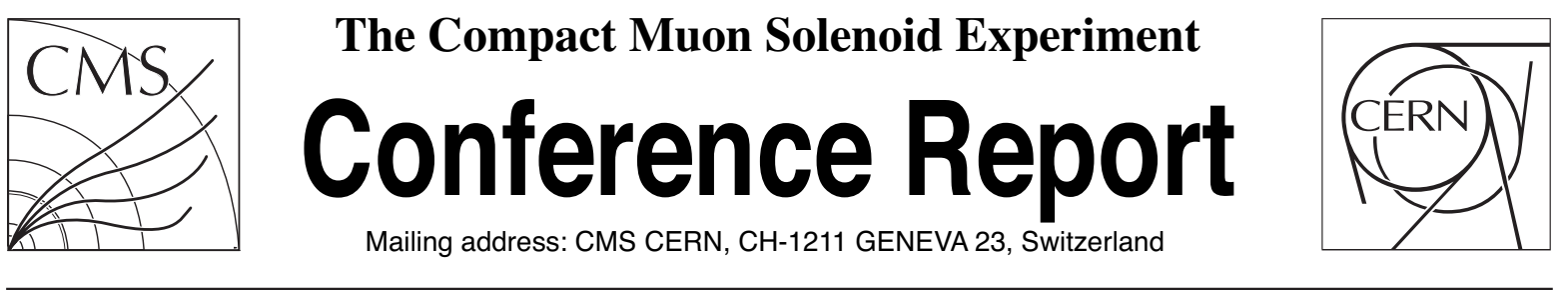

09 October 2016 (v2, 14 October 2016)

\title{
The Phase1 CMS Pixel detector upgrade
}

\author{
Vittorio Raoul Tavolaro for the CMS Collaboration
}

\begin{abstract}
The pixel detector of the CMS experiment will be replaced in an extended end-of-year shutdown during winter 2016/2017 with an upgraded one able to cope with peak instantaneous luminosities beyond the nominal LHC instantaneous luminosity of $1 \times 10^{34} \mathrm{~cm}^{-2} \mathrm{~s}^{-1}$. Under the conditions expected in the coming years, which will see an increase of a factor two in instantaneous luminosity, the present system would experience a dynamic inefficiency caused mainly by data losses due to buffer overflows. The Phase I upgrade of the CMS pixel detector, described in this paper, will operate at full efficiency at an instantaneous luminosity of $2 \times 10^{34} \mathrm{~cm}^{-2} \mathrm{~s}^{-1}$, thanks to a new readout chip. The new detector will feature one additional tracking point both in the barrel and in the forward regions, while reducing the material budget as a result of a new $\mathrm{CO}_{2}$ cooling system and optimised layout of the services. In this paper, the design and the technological choices of the Phase I detector will be reviewed and the status of the construction of the detector and the performance of its components will be discussed.
\end{abstract}

Presented at PIXEL2016 International Workshop on Semiconductor Pixel Detectors for Particles and Imaging. Deadline for submission: 28 October 2016 


\section{The Phase1 CMS Pixel detector upgrade}

\section{Vittorio Raoul Tavolaro on behalf of the CMS collaboration}

Institute for Particle Physics, Eidgenössische Technische Hochschule Zürich, Switzerland.

E-mail: vittorio.tavolaro@cern.ch

Aвstract: The pixel detector of the CMS experiment will be replaced in an extended end-of-year shutdown during winter 2016/2017 with an upgraded one able to cope with peak instantaneous luminosities beyond the nominal LHC instantaneous luminosity of $1 \times 10^{34} \mathrm{~cm}^{-2} \mathrm{~s}^{-1}$. Under the conditions expected in the coming years, which will see an increase of a factor two in instantaneous luminosity, the present system would experience a dynamic inefficiency caused mainly by data losses due to buffer overflows. The Phase I upgrade of the CMS pixel detector, described in this paper, will operate at full efficiency at an instantaneous luminosity of $2 \times 10^{34} \mathrm{~cm}^{-2} \mathrm{~s}^{-1}$, thanks to a new readout chip. The new detector will feature one additional tracking point both in the barrel and in the forward regions, while reducing the material budget as a result of a new $\mathrm{CO}_{2}$ cooling system and optimised layout of the services. In this paper, the design and the technological choices of the Phase I detector will be reviewed and the status of the construction of the detector and the performance of its components will be discussed.

Keywords: Radiation-hard detectors, Si microstrip and pad detectors, Particle tracking detectors, Large detector systems for particle and astroparticle physics, Digital electronic circuits, Modular electronics, Radiation-hard electronics, Large detector-systems performance, Performance of High Energy Physics Detectors, Detector cooling and thermo-stabilization, Detector design and construction technologies and materials 


\section{Contents}

1 Introduction 1

2 Concept and design of the CMS Phase1 Pixel detector 2

3 Readout chip and pixel detector modules 3

4 Services 5

4.1 Cooling system 5

4.2 Power services 5

5 Structure and assembly $\quad 6$

$\begin{array}{lll}6 & \text { System tests } & 7\end{array}$

$\begin{array}{lll}7 & \text { Summary } & 7\end{array}$

\section{Introduction}

The innermost detector of the CMS experiment [1] at CERN is a hybrid pixel detector. It consists of $\mathrm{n}^{+}$-in-n silicon sensors segmented in pixels with a size of $100 \times 150 \mu \mathrm{m}^{2}$ in the $r-\phi$ and $z$ coordinates, respectively ${ }^{1}$. It features three layers in the barrel region (BPix) and two disks on each side of the forward region (FPix), with a total of 66 million pixels. This detector provided excellent performance, assuming a pivotal role in the tracking and vertexing measurements of CMS, during the Run 1 and 2 of the LHC. For example, the resolution in the barrel region, measured on data collected during 2015 at the center of mass energy of $13 \mathrm{TeV}$, is of the order of $10 \mu \mathrm{m}$ in the $r-\phi$ direction and of the order of $25 \mu \mathrm{m}$ in the $z$ direction. In 2015 the fraction of good channels was $98.6 \%$ and the hit efficiency was very high for all layers. More details on the performance of the present pixel detector are given in [2].

The present detector was designed to deliver such excellent performance at the nominal LHC instantaneous luminosity of $1 \times 10^{34} \mathrm{~cm}^{-2} \mathrm{~s}^{-1}$ with a bunch spacing of $25 \mathrm{~ns}$ and an average number of inelastic collisions per bunch crossing (pile-up) of 25. These conditions have been reached and exceeded by more than $20 \%$ during the current data taking period (Run 2) of the LHC, started in 2015, with the peak instantaneous luminosity foreseen to reach $2 \times 10^{34} \mathrm{~cm}^{-2} \mathrm{~s}^{-1}$ before 2018. Under such conditions, the present pixel system is expected to experience significant hit inefficiencies (16\% in the innermost layer), mainly due to buffer overflow in the present ReadOut

${ }^{1}$ CMS uses a right-handed coordinate system. The $x$-axis points to the center of the LHC ring, the $y$-axis points up vertically and the $z$-axis points along the beam direction. The azimuthal angle $\phi$ is measured in the $x y$-plane and the radial coordinate is denoted by $r$. The polar angle $\theta$ is defined in the $r z$-plane and the pseudorapidity is $\eta=-\ln \tan \frac{\theta}{2}$. 
Chip (ROC), which would cause a significant degradation of the physics performance of the CMS detector.

The following section gives an overview of the concept and the design of the Phase 1 upgrade of the CMS pixel detector. The design and the improvements of new readout chip are discussed in Section 3, together with the discussion of design and production of pixel detector modules. Section 4 reviews the powering and cooling systems of the upgraded detector, while in Section 5 the mechanical structure and assembly of the detector are discussed in detail. System tests where operation of the upgrade detector is exercised are reviewed in Section 6.

\section{Concept and design of the CMS Phase1 Pixel detector}

The Phase1 upgrade of the pixel detector will allow to maintain and improve the performance of the present detector in the harsh conditions of the coming years, being fully efficient and providing robust tracking up to instantaneous luminosities of $2 \times 10^{34} \mathrm{~cm}^{-2} \mathrm{~s}^{-1}$. The hit inefficiencies are reduced thanks to a new improved ROC with faster readout and increased buffers.

The Phase1 Upgrade pixel detector shares several aspects with the present system, while at the same time it introduces considerable improvements and novelties. This approach allows to exploit the knowledge acquired during design, construction and operation of the present system and apply it to the improved new system, allowing to limit the research and development phase of the project considerably.

The geometrical layout of the upgrade system consists of four cylindrical barrel layers placed at radii of 29, 68, 109, $160 \mathrm{~mm}$ and three disks in each of the forward regions placed at a distance from the nominal interaction point of 291, 396 and $516 \mathrm{~mm}$. This layout is optimised in order to offer full 4-hit tracking coverage up to pseudorapidities of 2.5, with an increased redundancy compared to the present system. The layout of the Phase 1 detector is compared with the present one in figure 1. The installation of a new beampipe during 2014 with a smaller radius of $45 \mathrm{~mm}$ enables the placement of the innermost barrel layer closer to the interaction point in the upgrade detector $(29 \mathrm{~mm})$ compared to the present detector $(44 \mathrm{~mm})$, with great benefit for vertex resolution and b-tagging efficiency. The fourth barrel layer extends the pixel detector tracking volume closer to the CMS silicon strip tracker system, improving the overall tracking performance of the experiment by reducing the combinatorics in track reconstruction.

The total number of pixels increases from 66 millions to 124 millions, while at the same time the material budget is reduced. The amount of material of the upgraded detector is comparable to the one of the present detector in the central region $(|\eta| \lesssim 1.2)$, despite the addition of an extra detector layer, and it is significantly reduced at higher pseudorapidities. This is achieved thanks to new light-weight support structures and to a new $\mathrm{CO}_{2}$ evaporative cooling system, replacing the present $\mathrm{C}_{6} \mathrm{~F}_{14}$ mono-phase cooling system, and thanks to an optimised layout of the services. The new detector will employ a new $\mu$ TCA-based DAQ, replacing the present VME-based DAQ.

Both the Phase1 and the present system are built following a modular detector concept. A total of 1184 silicon sensor modules constitute the BPix layers, while 672 are used for the FPix disks. The detector modules comprise, as in the present detector, a $285 \mu \mathrm{m}$ thick silicon sensor, with a $n^{+}$-in- $n$ technology, segmented with a cell size of $100 \times 150 \mu \mathrm{m}^{2}$. 


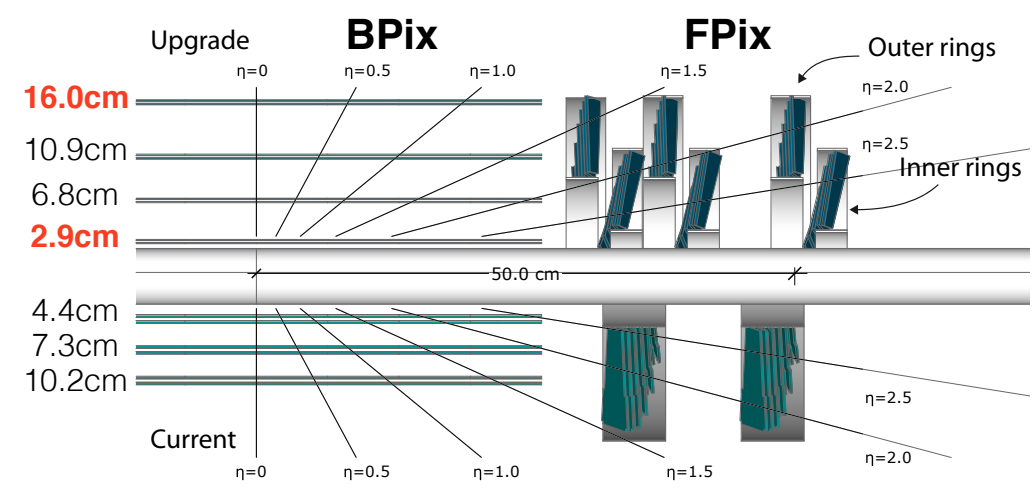

Figure 1: Comparison of geometrical layouts of the current (bottom) and upgrade (top) CMS pixel detectors.

\section{Readout chip and pixel detector modules}

The readout chip of the upgrade detector, named psi46digv21respin, is based on the present psi46 readout chip [3]. The same process technology $(250 \mathrm{~nm}$ CMOS) is employed in the new chip, as well as the zero-suppressed readout architecture based on the column drain mechanism. The chip readout is segmented into 4160 pixels, arranged in an array of 80 rows and 26 double-columns, covering an area of $102 \times 79 \mathrm{~mm}^{2}$. Several important improvements are introduced in the upgrade chip:

- the present $40 \mathrm{MHz}$ analog readout is replaced by a $160 \mathrm{Mbits} / \mathrm{s}$ digital readout, adding an additional 8-bit ADC to format the pulse-height information;

- the hit and timestamp buffer sizes have been significantly increased, from 32 to 80 and from 12 to 24 , respectively;

- the radiation-hardness of the chip is improved, by optimising the transistor type choice in critical points of the design and by introducing enlarged DAC ranges that allow to compensate for more extreme radiation-induced effects;

- an improved comparator and a reduced chip-internal cross-talk lead to an operational threshold of $\sim 1800 \mathrm{e}^{-}$with a noise of $\sim 100 \mathrm{e}^{-}$, much lower compared to the threshold of $\sim 3500$ $\mathrm{e}^{-}$of the present chip. This improves both the efficiency and the resolution of the detector, while increasing its longevity.

The radiation tolerance of the chip has been tested after irradiation with $23 \mathrm{MeV}$ protons at the ZAG Cyclotron in Karlsruhe. A dose of about $0.6 \mathrm{MGy}$ is expected to be accumulated by the second innermost barrel layer (L2) of the upgrade detector, after $\sim 500 \mathrm{fb}^{-1}$ of proton-proton data have been collected. The chip has been tested after nominal exposure to 0.6, 1.2 (expected innermost layer (L1) dose after $500 \mathrm{fb}^{-1}$ ) MGy and to higher doses of 2.4 and 4.8 MGy. The pixel thresholds could be aligned and set to $\sim 1800 \mathrm{e}^{-}$even after the highest dose exposure, while keeping the noise under $180 \mathrm{e}^{-}$. The hit efficiency at the expected L2 hit rate shows no degradation after the two realistic dose levels of 0.6 and $1.2 \mathrm{MGy}$. 
The sources of inefficiency in the chip readout have been simulated and validated with singlehit X-ray events, showing excellent agreement with measurements. The same simulation is used to simulate LHC-like conditions ( 2.3 hits/timestamp) and shows that inefficiencies are below $2 \%$ at the expected L2 hit-rate, but become significantly larger at the expected L1 hit-rate, in accord with predictions. The innermost layer will indeed use a further evolution of this chip design, with an improved readout mechanism of groups of $2 \times 2$ pixels, better radiation-hardness and capability to withstand rates up to $600 \mathrm{MHz} / \mathrm{cm}^{2}$. The chip behaviour has been thoroughly verified and tested in beam test setups. The details of these studies are presented in [4].

The detector module structure is largely similar to the one employed in the present detector. Figure 2 shows exploded views of L1 and L2-4 modules.
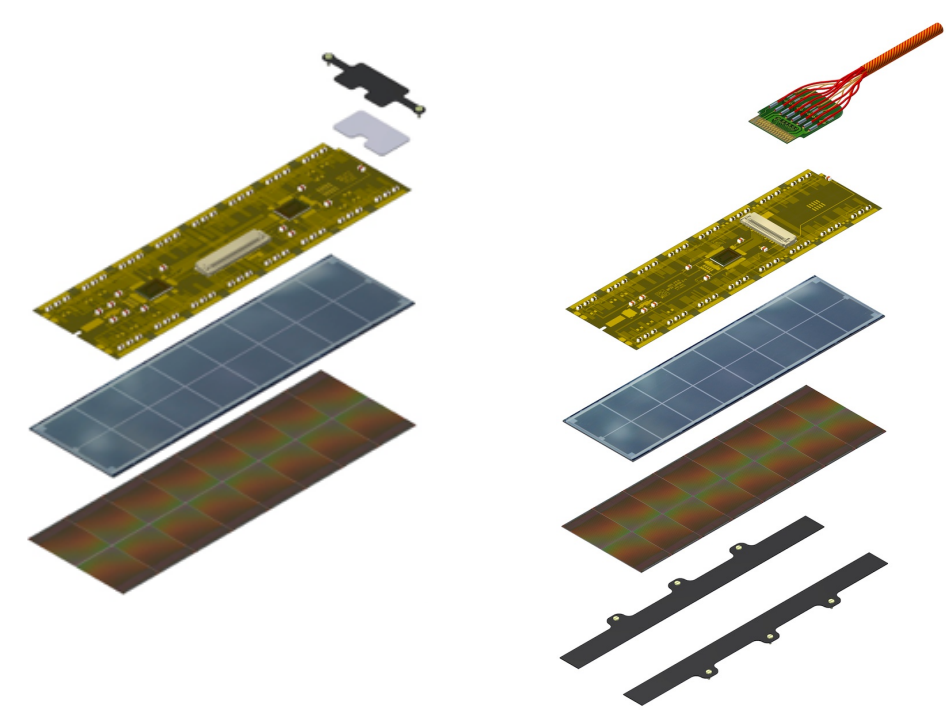

Figure 2: Exploded views of L1 (left) and L2-4 (right) modules.

The core of the module is the $285 \mu \mathrm{m}$ thick $n^{+}$-in- $n$ silicon sensor, with an active area of $16.2 \times 64.8 \mathrm{~mm}^{2}$, which is bump-bonded to an array of $2 \times 8$ ROCs. A thin PCB called HighDensity Interconnect (HDI) is glued to the back-plane of the sensor and wire-bonded to the chips, distributing signals and power to all chips. A token-bit manager (TBM), glued and wire-bonded on top of the HDI, supervises the token chain ring of the chips and groups readouts of ROCs onto $320 \mathrm{MHz}$ links (400 MHz after 4/5 bit encoding). Due to the higher rates and the larger required bandwidth, two TBMs are installed on L1 modules. The module is connected to the electronic boards and power through a $1 \mathrm{~m}$ long micro-twisted pair low-mass cable. L2-4 modules are glued to $\mathrm{Si}_{3} \mathrm{~N}_{4}$ base strips, which provide mechanical stability and are used for fixing the modules onto the final structure. L1 modules are instead clamped using carbon fiber clips on the short side of the module, further reducing the material budget of the innermost layer. The FPix modules differ in a few details (e.g. the cable), but they share the same concept and geometry, employing the L2-4 ROC.

Assembled modules are tested and calibrated in order to identify faulty modules and discard them prior to the installation. Dedicated setups reproducing environmental conditions of the final system are used to carry out module qualification. The modules undergo a series of functionality 
tests and calibrations at low temperature $\left(-20^{\circ} \mathrm{C}\right)$, before and after a series of thermal cycles, and at room temperature $\left(+17^{\circ} \mathrm{C}\right)$. The sensor is also characterised through a measurement of its leakage current as a function of the bias voltage (IV measurement), performed both at low and room temperature. A grading system is developed in order to automatically divide modules into three classes, the last one being the discarded modules, based mainly on pixel defects and sensor IV. Modules are also tested in X-ray setups. The X-ray beam is used to generate intense readout traffic in the ROCs and to measure single-hit efficiency and other properties in those conditions. The ROC energy-response is also measured using fluorescence lines as reference.

A total of 1184 modules are needed to build the BPix part of the detector. The amount of modules needed for the construction of L2-4 has been reached during summer 2016 and the production is going on through fall 2016 to produce the desired amount of spare modules. L1 modules are being produced at the time of writing this paper. The relative fraction of L1 modules is relatively small (96 modules, $8 \%$ of total BPix production) and the production is expected to be completed at the beginning of November 2016. The FPix system comprises 672 modules and their production is approaching completeness. The yields of module production for the different layers and for FPix line up at $\sim 80 \%$.

\section{Services}

\subsection{Cooling system}

The upgrade detector is cooled by a new bi-phase $\mathrm{CO}_{2}$ system, replacing the current mono-phase $\mathrm{C}_{6} \mathrm{~F}_{14}$ liquid cooling. The cooling tubes are arranged in complex looping structures and run along both the detector mechanical structure, in which they are embedded, and the structures housing the services. The stainless steel cooling tubes are much thinner compared to the present system, with an inner diameter of $1.6 \mathrm{~mm}$ and a wall thickness between 50 and $100 \mu \mathrm{m}$, strongly contributing to the reduction of the material budget of the upgrade detector. The system is operated at high pressure $\left(20 \mathrm{bar}\right.$ ) at a nominal temperature of $-20^{\circ} \mathrm{C}$, with the possibility of cooling the detector to lower temperatures. The typical heat load removed by one loop is $200 \mathrm{~W}$. All cooling loops for the final system have been produced, cut and bent and are now being laser-welded to produce the final structure. The production and the embedding in the mechanical support structure of the cooling system for L1 and L2 is complete and the support structures are ready for module mounting.

\subsection{Power services}

The upgrade detector features 1.9 times more channels compared to the present detector, and its current consumption increases thus almost four times. Nevertheless, it will be powered using the existing power supplies, which will not be replaced in this upgrade project. The upgrade system uses a DC-DC powering scheme to supply the necessary power to the modules. The power is transferred using $\sim 40 \mathrm{~m}$ long low-voltage lines from the power supplies to the DC-DC converters, installed on service structures at $|\eta| \approx 4$. The converters receive $10 \mathrm{~V}$ that are converted to 3.0/2.4 $\mathrm{V}$ powering the digital and analog circuits of the modules, respectively. The fixed output voltage of the converters has been optimised taking into account the module cable lengths and the impact of radiation on the powering requirements of the modules. The DC-DC converters are based on the 
FEAST2 ASIC and customised for this application. In total 1184 converters are needed to power the detector and their production is complete.

\section{Structure and assembly}

The detector modules are mounted on lightweight mechanical structures. The BPix mechanical structure is built of a Carbon Fiber Reinforced Polymer (CFRP) and Airex foam compound, characterised by a high thermal conductivity. A prototype of such structure for the L1 is shown in Figure 3a. It is built in half cylinders and it is organised in support strips, in which rectangular cut-outs are machined to minimise its impact on the total material budget. Each half cylinder is supported by an end-flange, where the routing of the module cables is organised and implemented. The construction of the BPix structure is well advanced: the half cylinders for the two innermost layers (L1 and L2) are fully assembled and integrated with cooling pipes, while the structures of the two outermost ones (L3 and L4) are in preparation. The BPix structure is supported by and connected to the so-called BPix "supply tube", also built in half shells. It hosts several types of electronics boards for signal distribution and readout, Opto-Hybrid converters for electro-optical conversion and DC-DC converters for powering. It also carries the services such as cooling pipes and optical fibers. The location of the electronic boards has been optimised in the new system, shifting them to larger pseudorapidities and thus reducing the material budget in the active tracking volume.

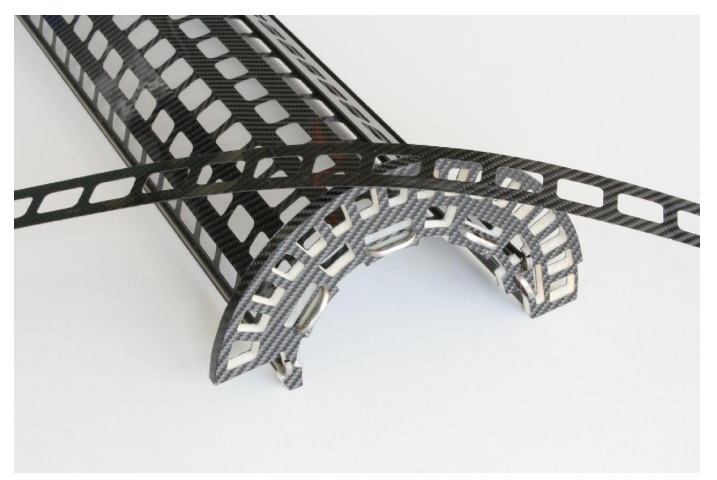

(a) Prototype of the BPix L1 mechanical structure.
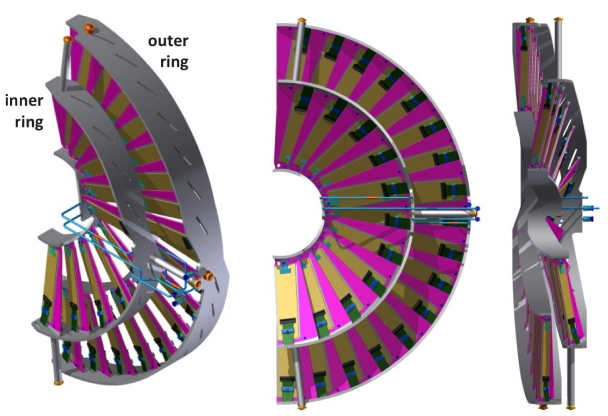

(b) The FPix disk layout.

Figure 3: The mechanical structures of BPix and FPix regions.

The FPix mechanical structure is also organised in half cylinders (two for each side), hosting the electronics boards and services, where the disks are housed. Each disk comprises an inner and an outer graphite ring, arranged concentrically as shown in Figure 3b, where the modules are glued with thermal film onto structures of Thermal Pyrolitic Graphite (TPG). The cooling tubes are embedded in the rings. The first two FPix half-cylinders, including disks equipped with final detector modules, have been fully assembled and integrated. Tests of the system integrated with $\mathrm{CO}_{2}$ and with new $\mu$-TCA readout are successful, showing $>99.98 \%$ responsive channels. The 
first half cylinder has been disassembled and shipped to CERN in view of the final installation and the second one is expected by the end of September 2016. The preparation of the third and fourth half-cylinders is advancing well.

\section{System tests}

System tests are developed in order to test the performance of the full chain of components of the upgrade final system and to exercise its operation. Test stands are set up at University of Zurich for BPix and at FNAL and CERN for FPix. They include close-to-final prototypes of all components of the detector: detector modules, control and readout electronics, CMS $\mu$ TCA DAQ systems, power and cooling system. The main goal is to establish and practice operation and calibration procedures prior to the installation of the full detector. The results are very promising and will provide a head start for the control and readout of the upgrade detector.

\section{Summary}

The CMS pixel system will be replaced by an upgrade detector in an extended year-end shutdown of the LHC starting at the end of 2016, with minimal impact on data taking. The new system features an additional tracking point in the whole tracking volume, while at the same time reducing its material budget. It will be able to operate at significantly higher rates and perform excellently at the increasing instantaneous luminosities of the LHC in the coming years, up to $2 \times 10^{34} \mathrm{~cm}^{-2} \mathrm{~s}^{-1}$ thanks to an improved readout chip. It employs a new light-weight $\mathrm{CO}_{2}$ cooling system and a DC-DC powering scheme. The module production is close to completion as well as the production, assembly and test of all final components. Operation and calibration of the new detector is established and exercised on test stands which include all final system components.

\section{References}

[1] CMS collaboration, The CMS experiment at the CERN LHC, 2008, JINST 3 S08004.

[2] Jànos Karancsi, Operational Experience with the CMS Pixel Detector, these proceedings.

[3] H.Chr. Kaestli et al., Design and Performance of the CMS Pixel Detector Readout Chip, Nucl. Instrum. Meth. A565 (2006) 188-194.

[4] Andrey Starodumov, High rate capability and radiation tolerance of the new CMS pixel detector readout chip PROC600, these proceedings. 